\title{
Functional genetic variants in the 3'UTR of PTPRD associated with the risk of gestational diabetes mellitus
}

\author{
YAN KANG, HUAMIN HUANG, HAIPENG LI, WENPING SUN and CUICUI ZHANG \\ Department of Obstetrics and Gynecology, Qinghai Red-Cross Hospital, Xining, Qinghai 810000, P.R. China
}

Received April 6, 2020; Accepted October 20, 2020

DOI: $10.3892 /$ etm.2021.9994

\begin{abstract}
A previous study revealed that protein tyrosine phosphatase receptor type D (PTPRD) is highly associated with diabetes mellitus, particularly for type 2 diabetes, through a genome-wide association study. However, the influence of the human polymorphism in the 3'-untranslated region (3'-UTR) of PTPRD on gestational diabetes mellitus (GDM) has remained to be defined. The present study focused on the functional polymorphism located in the 3'-UTR of PTPRD and whether it is associated with the susceptibility to develop GDM. A total of 1,100 pregnant female patients aged between 28 and 36 years within gestational weeks 24-28 were recruited. The participants enrolled in the study comprised 500 cases of GDM and 600 normal controls. Based on the screening results, the single nucleotide polymorphism (SNP) rs56407701 exhibited the most significant difference and may increase the susceptibility to GDM. A prediction of target microRNAs (miRNAs/miRs) using the miRNA SNP database indicated that SNP rs56407701 may be bound by miR-450a, causing the suppression of PTPRD expression in subjects with the GC or CC genotype. In conclusion, The CC genotype of PTPRD rs56407701, which may be bound by miR-450a, may increase the susceptibility of Chinese Han females to GDM during pregnancy. The present study provided a theoretical basis for the SNP rs56407701 being a source of GDM susceptibility loci.
\end{abstract}

\section{Introduction}

Gestational diabetes mellitus (GDM) is defined as any degree of abnormal glucose tolerance that occurs during pregnancy $(1,2)$. Glucose intolerance is diagnosed during pregnancy and GDM is one of the most common complications of pregnancy, affecting $3-8 \%$ of pregnancies (3). The

Correspondence to: Dr Yan Kang, Department of Obstetrics and Gynecology, Qinghai Red-Cross Hospital, 55 South Street, Xining, Qinghai 810000, P.R. China

E-mail: kangyan12344@yeah.net

Key words: genetics, microRNA-450a, 3'-untranslated region, glucose, protein tyrosine phosphatase receptor type D mechanisms of GDM remain to be fully elucidated. Binding insulin resistance and impaired insulin secretion lead to an underlying pathophysiology that may be influenced by interactions between genetic and environmental factors $(4,5)$. The prevalence of GDM has increased in recent decades due to the increased mean age of pregnant females and increased susceptibility to obesity (6). Human and animal studies suggested that type 2 diabetes mellitus (T2D) and GDM may have certain pathological changes in common, including insulin resistance, cellular dysfunction and insulin deficiency (7). Studies such as genome-wide association studies (GWASs) and single nucleotide polymorphism (SNP) strategies have identified numerous susceptibility genes associated with increased T2D sensitivity, including protein tyrosine phosphatase receptor type D (PTPRD) $(8,9)$.

PTPRD is a member of the PTP family (10). PTPs are considered to regulate signaling molecules in a variety of cellular processes, including cell growth, differentiation, mitotic cycle and oncogenic transformation (11). The extracellular domain contains the meprin-A antigen-PTP domain (12). Transformation of the PTPRD gene may lead to the replacement of the highly conserved residues in the second tyrosine phosphatase catalytic domain Thr1365-to-Met, which is highly associated with the pathogenesis of cancer $(13,14)$. Wang et al (15) determined that the mutant tyrosine phosphatase is a tumor suppressor gene that regulates cell pathways and may potentially be utilized for therapeutic intervention in colorectal cancer. In addition, Chen et al (16) reported that silencing of PTPRD was caused by DNA methylation in a mouse model of T2D and in patients, and was correlated with DNA (cytosine-5)-methyltransferase 1 expression. The difference of postprandial blood glucose in the PTPRD rs17584499 CT+TT genotype was significantly lower than that of the rs17584499 CC genotype (17).

MicroRNAs (miRNAs/miRs) are small non-coding RNA molecules consisting of 19-25 nucleotides that have been identified to have an important role in various human diseases (18). Studies have suggested that miRNA expression was tissue-specific and it has been reported that certain miRNAs were specifically expressed in the placenta (placental-specific miRNA). miRNAs negatively regulated the expression of target genes at the post-transcriptional level by binding to the 3'-UTRs of the target information RNA $(19,20)$. Increasing evidence proved that SNPs located at miRNA binding sites may lead to decreased or increased target mRNA translation. This 
dysfunction was associated with cancer susceptibility $(21,22)$. For instance, the CC genotype of cyclin-dependent kinase inhibitor 2B rs1063192 in the miR-323b-5p binding site may increase the susceptibility of pregnant Chinese Han females to GDM (23).

The present study focused on the SNPs in the 3'UTR of PTPRD by using the miRNASNP-v3 bioinformatics software (http://bioinfo.life.hust.edu.cn/miRNASNP/\#!/) with the loss-or-gain strategy (24). A total of 17 potential SNPs were obtained as candidate SNPs, which may be associated with certain miRNAs and with the pathogenesis of GDM. Based on this, the association between the allele distribution and the susceptibility of GDM was further investigated in a case-control study.

\section{Materials and methods}

Study subjects. The study plan was approved by the Ethics Committee of The Red Cross Hospital of Qinghai Province (Xining, China) and written informed consent was obtained from all participants prior to data collection. The subjects provided written informed consent prior to specimen collection. The study was approved by the ethics committee of Qinghai Red-Cross Hospital (Xining, China). All methods were in accordance with the approved guidelines. Subjects who had been diagnosed with diabetes, had taken drugs that affect glucose metabolism or had other pregnancy complications were excluded from the study. GDM was diagnosed according to the standards of the American Diabetes Association (25). Subjects with negative results of the $50-\mathrm{g}$ glucose stimulation test or a normal glucose tolerance test were used as controls. Demographic data were collected for all individuals, including age, body height and weight, and resting blood pressure at 24-28 weeks of gestation during the first pregnancy. The pre-pregnancy body mass index was calculated as [weight $(\mathrm{kg}) /$ height $\left(\mathrm{m}^{2}\right)$ ]. Routine laboratory evaluations were performed, including analysis of glycosylated hemoglobin (HbA1C), triglycerides, total cholesterol, low-density lipoprotein cholesterol and high-density lipoprotein (HDL) cholesterol. Blood glucose and serum insulin concentrations were measured at 0,60 and $120 \mathrm{~min}$ after the $100 \mathrm{-g}$ oral glucose tolerance test (OGTT). A total of 1,100 subjects were recruited, including 500 patients with GDM and 600 controls (Table I).

SNP selection. First, SNPs from the 3'-UTR of the PTPRD gene were obtained from the National Center for Bioinformatics (NCBI) SNP database (dbSNP; http://www. ncbi.nlm.nih.gov/snp/) and ENSEMBL v58 (https://www. ensembl.org/index.html). From the 1000 genome browser (http://www.1000genomes.org/), SNPs in the Chinese population were identified. Subsequently, the miRanda (http://www.microrna.org), TargetScan (http://www.targetscan. org/), PolymiRTS (http://compbio.uthsc.edu/miRSNP/) and miRDB software package (http://mirdb.org/) were used to study the miRNA targets of the SNPs of the binding sites. A bioinformatics analysis (http://bioinfo.life.hust.edu. cn/miRNASNP2/index.php) was used to determine the function of the miRNA targets of the SNPs of the 3'-UTR of PTPRD. If the SNP was in a high linkage disequilibrium $\left(r^{2}>0.8\right)$, only one SNP was genotyped. For the gain-loss strategy analysis, the miRNA wild-type (wild) sequence and SNP allele sequence was obtained (24). Subsequently, two target prediction tools, TargetScan (http://www.targetscan. org/) and miRanda (http://www.microrna.org), were respectively used to estimate the target site. A total of four results were recorded, namely wild TargetScan (WT), wild miRanda (WM), SNP TargetScan (ST) and SNP miRanda (SM). If the target gene of a miRNA was present in both the WT and WM, but not in the ST and SM, the miRNA was considered to have a loss of this target gene. Conversely, if a target was present in both ST and SM, but not WT and WM, the miRNA was considered to have gained one target gene (26).

Genotyping. Genomic DNA was extracted from leukocyte microspheres of human plasma samples by traditional protease K (Beyotime Institute of Biotechnology) digestion and then extracted by phenol-chloroform and ethanol precipitation. The TaqMan SNP genotyping test was used for genotyping. PCR was performed in a total volume of $5 \mu \mathrm{l}$ containing TaqMan General Master Mix (Beyotime Institute of Biotechnology), 80X SNP genotyping Mix (Beyotime Institute of Biotechnology), DNase-free Water and $10 \mathrm{ng}$ genomic DNA. The PCR conditions were $2 \mathrm{~min}$ at $50^{\circ} \mathrm{C}$, $10 \mathrm{~min}$ at $95^{\circ} \mathrm{C}$ and 40 cycles at $95^{\circ} \mathrm{C}$ for $15 \mathrm{sec}$ and $60^{\circ} \mathrm{C}$ for $1 \mathrm{~min}$ in an ABI 7900HT Real-Time PCR system (Thermo Fisher Scientific, Inc.). The PCR products were visualized by $1 \%$ agarose gel electrophoresis using ethidium bromide (MilliporeSigma).

Cell lines and culture. The 293T cell line was purchased from the cell bank of the Chinese Academy of Sciences. Cells were cultured in RMI-1640 (Gibco; Thermo Fisher Scientific, Inc.) with $10 \%$ FBS (Invitrogen; Thermo Fisher Scientific, Inc.) in a humidified atmosphere with $5 \% \mathrm{CO}_{2}$ at $37^{\circ} \mathrm{C}$. miR-450a mimics (5'-TTTTTGCGATGTGTTCCTAAT G-3') and miR mimics control (5'-ACGUGACACGUUCGG AGAATT-3') were obtained from GenScript. Lipofectamine ${ }^{\circledR}$ 3000 (Invitrogen; Thermo Fisher Scientific, Inc.) was used for transfection in accordance with the manufacturer's protocol.

Reverse transcription-quantitative $(R T-q) P C R$ assay. Total RNA was obtained from placental tissues harvested after birth by using TRIzol ${ }^{\circledR}$ reagent as described by the manufacturer (Invitrogen; Thermo Fisher Scientific, Inc.). For mRNA detection, total RNAs (500 ng) were reverse transcribed using an RT kit (cat. no. D350A; Takara Bio, Inc.). GAPDH was used as an internal control. qPCR was performed using ABI Prism 7900HT (Applied Biosystems; Thermo Fisher Scientific, Inc.) according to the manufacturer's protocol of Mir-XTM miRNA qRT-PCR SYBR ${ }^{\circledR}$ kit (cat. no. 638314; Takara Bio, Inc.). The amplification conditions were $95^{\circ} \mathrm{C}$ for $10 \mathrm{~min}$, followed by 40 cycles of $95^{\circ} \mathrm{C}$ for $30 \mathrm{sec}, 55^{\circ} \mathrm{C}$ for $40 \mathrm{sec}$ and $72^{\circ} \mathrm{C}$ for $30 \mathrm{sec}$, and finally $4^{\circ} \mathrm{C}$ for $30 \mathrm{~min}$ for cooling. The primers used were as follows: PTPRD forward, 5'-CTCCAAGGTTTA CACGAACACC-3'; and reverse, 5'-AGTCCGTAAGGGTTG TATTCTGA-3'; GAPDH forward, 5'-GGAGCGAGATCC CTCCAAAAT-3'; and reverse, 5'-GGCTGTTGTCATACT TCTCATGG-3'. The results were assessed using the $2^{-\Delta \Delta C q}$ method $(27,28)$. 
Table I. Frequency distributions of selected variables in patients with GDM and healthy controls.

\begin{tabular}{|c|c|c|c|}
\hline Variables & GDM (n=500) & Control $(n=600)$ & P-value \\
\hline Age (years) & $32 \pm 4$ & $32 \pm 3$ & 0.320000 \\
\hline Pregestational BMI $\left(\mathrm{kg} / \mathrm{m}^{2}\right)$ & $21.55 \pm 1.84$ & $21.61 \pm 1.92$ & 0.410000 \\
\hline Fasting plasma glucose $\mathrm{e}^{\mathrm{a}}(\mathrm{mmol} / \mathrm{l})$ & $5.02 \pm 0.78$ & $4.67 \pm 0.12$ & 0.000280 \\
\hline \multicolumn{4}{|l|}{ OGTT (mmol/l) } \\
\hline $0 \mathrm{~h}$ & $5.04 \pm 0.89$ & $4.50 \pm 0.11$ & 0.000350 \\
\hline $1 \mathrm{~h}$ & $10.66 \pm 1.25$ & $8.01 \pm 0.84$ & 0.000410 \\
\hline $2 \mathrm{~h}$ & $8.99 \pm 2.11$ & $7.02 \pm 0.34$ & 0.000521 \\
\hline Physical activity (number) & & & 0.000010 \\
\hline $0-150$ min per week & 341 & 201 & \\
\hline$\geq 150$ min per week & 159 & 399 & \\
\hline Glycosylated hemoglobin (\%) & $5.8 \pm 0.22$ & $4.7 \pm 0.12$ & 0.000320 \\
\hline Cholesterol (mmol/l) & $6.09 \pm 0.45$ & $6.08 \pm 0.33$ & 0.440000 \\
\hline Triglyceride (mmol/l) & $2.55 \pm 0.98$ & $2.45 \pm 0.63$ & 0.420000 \\
\hline HDL cholesterol (mmol/l) & $1.97 \pm 0.11$ & $2.01 \pm 0.21$ & 0.220000 \\
\hline LDL cholesterol (mmol/l) & $3.01 \pm 0.24$ & $3.07 \pm 0.28$ & 0.290000 \\
\hline
\end{tabular}

${ }^{\text {aF }}$ asting plasma glucose in the first trimester. A two-sided Student's t-test was used for either genotype distributions or allele frequencies between cases and controls, while for physical activity, the chi-square test was used. GDM, gestational diabetes mellitus; BMI, body mass index; H/LDL, high/low-density lipoprotein; OGTT, oral glucose tolerance test.

Construction of luciferase-based reporter plasmids. The fragments containing the 3'-UTR with the G or C alleles of SNP rs56407701 were amplified by GenScript. The PCR product was cloned into the pMIR-REPORT luciferase system (Ambion; Thermo Fisher Scientific, Inc.). The amplified fragment was verified by DNA sequencing by GenScript. The pRL-TK vector containing Renilla luciferase was used as a normalization control.

Dual-luciferase reporter assay. The 3'-UTR sequence of PTPRD predicted to interact with miR-450a or the mutant sequence of the predicted target (synthesized by GenScript) were inserted into the pMIR-REPORT vector at the restriction enzyme cutting site of HindIII and SacI (provided by GenScript). 293T cells $\left(1 \times 10^{5}\right)$ cultured on 24-well plates were co-transfected with pMIR reporter vectors containing wild-type or mutant PTPRD 3'-UTR fragments and miR-450a or control, and pRL-TK containing Renilla luciferase was used for normalization.

Statistical analysis. Data were examined regarding whether they followed a normal distribution in order to select an appropriate parametric or non-parametric test. The chi-square test was applied to compare differences in categorical variables. The skewness coefficient and kurtosis coefficient were determined by 0 and the Kolmogorov-Smirnov test was performed by $\mathrm{P}>0.05$. For continuous variables with a normal distribution, the results were expressed as the mean \pm SD or the mean \pm SEM. For continuous variables with a normal distribution, an unpaired Student's t-test was used to compare between two groups. For comparison of multiple groups, ANOVA was applied. In the case of significant results obtained by ANOVA, Bonferroni's post hoc test was employed as a multiple-comparisons test with 0.05 as the significance level. The Hardy-Weinberg equilibrium for each SNP genotype was determined by the chi-square test. The relationship between SNPs and the susceptibility to GDM was calculated by multivariate logistic regression analysis with the odds ratio (OR) and $95 \%$ CI. All statistical tests were bilateral and $\mathrm{P}<0.05$ was considered to indicate statistical significance.

\section{Results}

Patient characteristics. The clinical characteristics of patients with GDM and the control group are presented in Table I. No difference was obtained in age and gender distribution. After fasting and in the OGTT, blood glucose, HbA1C and triglycerides of patients with GDM were higher than those in the control group $(\mathrm{P}<0.001)$. The HDL cholesterol level in the GDM group was significantly lower than that in the control group $(\mathrm{P}<0.05)$. Sufficient physical activity during pregnancy was associated with a significantly reduced incidence of GDM.

Although the original rationale of the present study was focused on genetics, according to a recent report the food intake was also associated with GDM (29). Differences in food intake between pregnant females with and without GDM, including cereals, vegetables, fruit, dairy products and sweet beverages, were also analyzed. Furthermore, ORs and respective 95\% CIs of developing GDM between the two categories of food consumption were calculated, i.e. 'once a day or more' vs. 'less than once a day'. Regarding daily consumption, females with GDM exhibited a significantly more frequent daily intake of dairy products and sweet beverages (Table SI).

miR SNPs in the 3'-UTR of the PTPRD gene and genotype and allele analysis. As presented in Table II, 17 SNPs were 
Table II. SNPs located in the 3'-UTR of the protein tyrosine phosphatase receptor type D gene and the predicted miRNAs.

\begin{tabular}{|c|c|c|c|c|}
\hline SNP & Chromosome & 3'-UTR position & Associated miRNA & Allele \\
\hline rs62536166 & 9 & $3007-3030$ & $\mathrm{miR}-148 \mathrm{a} / 148 \mathrm{~b} / 152 / 153$ & $\mathrm{~A} / \mathrm{C}$ \\
\hline rs77547574 & 9 & $2348-2371$ & miR-515/1283 & $\mathrm{C} / \mathrm{A}$ \\
\hline rs73428138 & 9 & $761-782$ & miR-548/1323 & $\mathrm{C} / \mathrm{T}$ \\
\hline rs117224071 & 9 & $1419-1441$ & miR-802 & $\mathrm{A} / \mathrm{G}$ \\
\hline rs77185985 & 9 & $2682-2703$ & $\operatorname{miR}-759 / 2673$ & $\mathrm{~T} / \mathrm{G}$ \\
\hline rs114870484 & 9 & $2290-2312$ & $\operatorname{miR}-942 / 3182$ & $\mathrm{C} / \mathrm{G}$ \\
\hline rs73428138 & 9 & $760-782$ & $\mathrm{miR}-548 \mathrm{e} / \mathrm{f} / \mathrm{o} / \mathrm{t} / 3609$ & $\mathrm{C} / \mathrm{T}$ \\
\hline rs10976945 & 9 & $1020-1037$ & miR-4311/3688/31 & $\mathrm{A} / \mathrm{C}$ \\
\hline rs1064270 & 9 & $444-464$ & miR-3679/4313 & $\mathrm{C} / \mathrm{T}$ \\
\hline rs56407701 & 9 & $3073-3095$ & miR-450a & $\mathrm{G} / \mathrm{C}$ \\
\hline rs116361362 & 9 & $435-454$ & miR-409 & $\mathrm{A} / \mathrm{G}$ \\
\hline rs75115513 & 9 & $821-842$ & miR-1321/548o & $\mathrm{A} / \mathrm{T}$ \\
\hline rs117795823 & 9 & $1676-1695$ & miR-670 & $\mathrm{C} / \mathrm{T}$ \\
\hline rs74775961 & 9 & $2596-2617$ & miR-1298 & $\mathrm{A} / \mathrm{G}$ \\
\hline rs28554480 & 9 & $1157-1173$ & miR-3201 & $\mathrm{A} / \mathrm{G}$ \\
\hline rs11542527 & 9 & $1576-1597$ & miR-3606 & $\mathrm{A} / \mathrm{G}$ \\
\hline rs79554842 & 9 & $819-842$ & miR-3613 & $\mathrm{A} / \mathrm{C}$ \\
\hline
\end{tabular}

Chr, chromosome; UTR, untranslated region; miRNA/miR, microRNA; SNP, single nucleotide polymorphism.

Table III. Genotype frequencies of the protein tyrosine phosphatase receptor type D rs56407701 polymorphism among GDM cases and controls.

\begin{tabular}{|c|c|c|c|c|}
\hline Genotype & GDM $(n=500)$ & Controls $(n=600)$ & OR $(95 \% \mathrm{CI})^{\mathrm{a}}$ & P-value ${ }^{a}$ \\
\hline GG & $411(82.2)$ & $522(87.0)$ & 1.00 & \\
\hline GC & $54(10.8)$ & $63(10.5)$ & $1.09(1.02-1.72)$ & 0.040 \\
\hline $\mathrm{CC}$ & $35(7.0)$ & $15(2.5)$ & $3.93(1.11-3.94)$ & 0.002 \\
\hline C carrier & $89(17.8)$ & $78(13.0)$ & $1.58(1.12-1.63)$ & 0.027 \\
\hline
\end{tabular}

${ }^{\mathrm{a} O R s,}$ 95\% CIs and P-value were calculated after adjusting for fasting plasma glucose, OGTT, physical activity and glycosylated hemoglobin. P-values are presented vs. GG only. OR, odds ratio; GDM, gestational diabetes mellitus.

predicted that may be bound by candidate miRNAs. Further genotyping was performed to identify the 17 SNP alleles.

First, the influence of the 17 candidate recognition SNPs on GDM susceptibility in cases vs. control subjects was explored. The results suggested that GDM was only associated with SNP rs56407701. As presented in Tables III and SII, chi-square statistical analysis indicated that the rs56407701 genotype exhibited a Hardy-Weinberg equilibrium pattern in healthy controls $(\mathrm{P}=0.22)$. Logistic regression analysis revealed that the susceptibility of the GC and CC genotypes to GDM was significantly higher than that of the GG genotype $(\mathrm{OR}=1.09$, 95\% CI, 1.02-1.72; OR=3.93, 95\% CI, 1.11-3.94, respectively). All ORs were adjusted for fasting plasma glucose, OGTT, physical activity and glycosylated hemoglobin.

miR-450a binds to the 3'UTR of PTPRD with the $C$ allele. The potential binding site of miR-450a in the 3'UTR of PTPRD predicted by the bioinformatics analysis is presented in Fig. 1A. According to this, in patients with the CC genotype, the binding ability was comparatively greater than in the other subjects with more binding sites. It was hypothesized that the expression of PTPRD may be regulated by miR-450a, which may be impacted by the SNP rs56407701. To confirm this, the expression of PTPRD mRNA expression in the placenta samples of the GDM patients mentioned above was further investigated. As presented in Fig. 1B, patients with GDM of the CC genotype had suppressed expression of PTPRD mRNA compared with those of the GC and GG genotype. Next, vectors including the allele-specific binding sequences were constructed and then co-transfected with miR-450a or the respective controls into the $293 \mathrm{~T}$ cell line. As presented in Fig. 1C, the luciferase reporter assay confirmed that miR-450a was able to bind with the 3'UTR of PTPRD with the CC genotype of the rs56407701 SNP resulting in the suppression of luciferase activity, which indicated that PTPRD expression was suppressed. However, the binding ability was abolished with the GG genotype of the rs56407701 SNP (wild-type). 
A

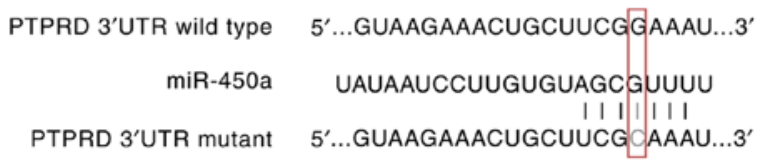

B

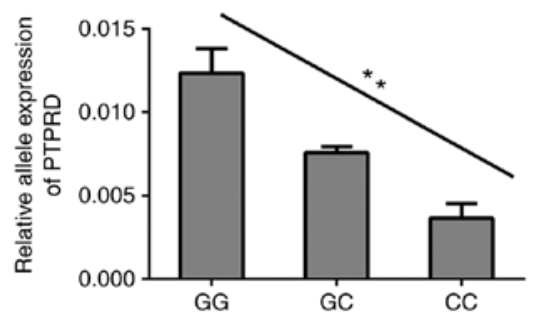

D

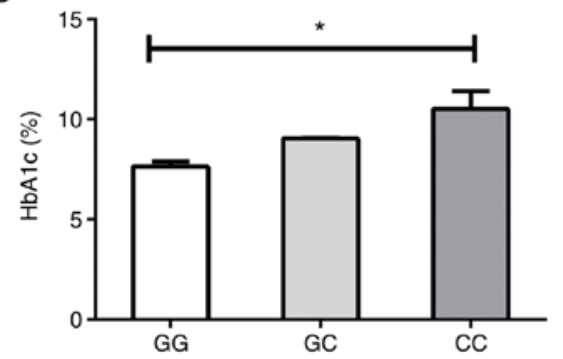

C
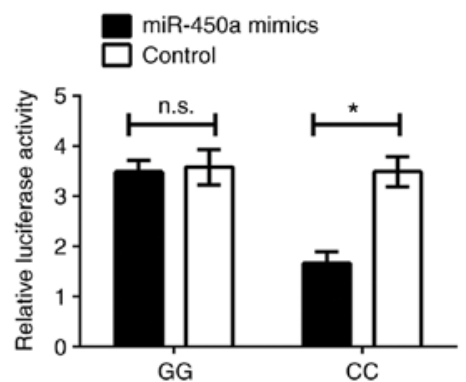

E

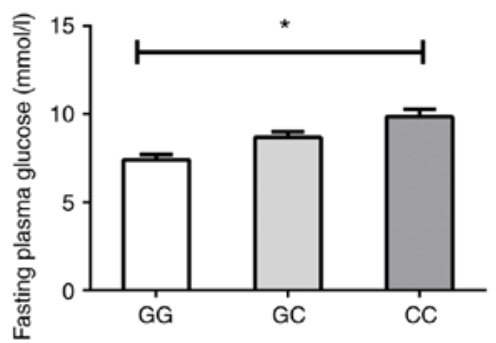

Figure 1. The miR-450a binding site is abolished by a single nucleotide polymorphism located in the 3'UTR of PTPRD. (A) The potential binding site of miR-450a in the 3'UTR of PTPRD, with G-allele distribution at the top and C-allele distribution at the bottom. (B) Relative expression levels of PTPRD in the GG, GC and CC genotypes were detected by reverse transcription-quantitative PCR in placental tissues. (C) Relative luciferase activity obtained with the pMIR-REPORT expression vector containing the GG genotype or CC genotype PTPRD 3'UTR region and transfected control or miR-450a. Renilla control vector was used as a normalization control and the ratio of Renilla/firefly luciferase activity of cells was calculated. (D) Percentage of HbA1c in GDM patients with different genotypes. (E) Fasting plasma glucose in patients with GDM with different genotypes. Values are expressed as the mean \pm standard error of the mean. ${ }^{*} \mathrm{P}<0.05$ and ${ }^{* *} \mathrm{P}<0.01$ vs. all groups. n.s., no significance. UTR, untranslated region; HbA1c, glycated hemoglobin; GDM, gestational diabetes mellitus; miR, microRNA; PTPRD, protein tyrosine phosphatase receptor type D.

Since it is widely known that HbAlc is closely associated with GDM (30), information regarding the plasma levels of HbAlc as well as the fasting plasma glucose levels of the patients was collected. These values were compared between patients with different genotypes of the rs56407701 SNP of PTPRD. As presented in Fig. 1D and E, GDM patients with the $\mathrm{C}$ allele exhibited higher HbA1c and fasting plasma glucose levels compared with both GG and GC groups.

\section{Discussion}

GDM is a complex disease caused by the combination of genetic factors and environmental exposure (31). miR-binding SNPs may serve as novel targets or destroy existing recognition sites, promoting disease susceptibility and important disease characteristics, particularly in cancer and human GDM (32). In the present study, the relationship between miR SNPs in the 3'-UTR of PTPRD and GDM susceptibility was investigated. It was observed that PTPRD rs56407701 was significantly associated with increased susceptibility and the SNP was located at the binding site of miR-450, interfering with the inhibition of PTPRD expression by miR-450, which had an important role in the occurrence and development of GDM.

To date, no direct evidence has been provided to support the role of miR-450a in GDM. Abnormal miRNA expression is associated with a variety of diseases. Research has identified the potential function of miR-450a in human disease. Upregulated miR-450a eliminated methylglyoxal-induced insulin resistance via targeting cyclic AMP response element binding protein and may therefore be used as a potential target to improve insulin resistance and treat patients with diabetes-associated diseases (33). Furthermore, after glutamine discontinuation, miR-450a overexpression decreased the mitochondrial membrane potential but increased glucose uptake and cell viability, which are characteristic of less invasive cancer cells. In summary, by regulating glutamine decomposition-associated targets, miR-450a may reduce the production of lipids, amino acids and nucleic acids and finally influence in the development of diabetes (34).

PTPs are signaling molecules that regulate a variety of cellular processes, including differentiation, cell proliferation, mitotic cycle and oncogenic transformation (35). PTPRD, which is associated with T2D and involved in the insulin signaling pathway, was first reported in a GWAS a Chinese Han population (36). Tsai et al (36) indicated that the PTPRD gene was related to T2D susceptibility in a Chinese Han population. Subsequently, Below et al (37) performed another GWAS with 837 T2D cases and 436 normoglycemic controls, followed by a meta-analysis, revealing such an association with another SNP, rs649891, in PTPRD in Mexican-Americans $(17,36)$. In a replication study, the 
PTPRD genetic variant was suggested to be associated with progression to diabetes in Han Chinese, most likely through increased insulin resistance. Recently, Chen et al reported that the levels of PTPRD were significantly decreased in patients with T2D and that this protein is involved in the insulin signaling pathway. Chen et al (16) revealed that the rs10511544, rs10756026 and rs10809070 SNPs in PTPRD may contribute to a decreased susceptibility to GDM in Han Chinese subjects. The SNP rs17584499 located in PTPRD was reported to interact with the therapeutic efficacy of pioglitazone (38). In the present study, the role of the PTPRD rs56407701 polymorphism in human GDM was first investigated and the inhibition of PTPRD in human GDM by miR-binding of an SNP was first reported. PTPRD mRNA levels were also suppressed in patients with GDM. These results were similar to those of patients with T2D (16). Furthermore, PTPRD was reported to be involved in the insulin signaling pathway: STAT3, a well-known oncoprotein, was inactivated by PTPRD activation and STAT3 was overexpressed while PTPRD was inhibited (39).

The abnormal distribution of PTPRD polymorphisms in GDM in the present study suggested a strong relationship with the occurrence of GDM. GDM patients with The $\mathrm{C}$ allele had a higher risk of developing GDM in the presence of hyperglycemic factors than those with the $\mathrm{G}$ allele. In addition, patients who had taken in too much protein (above the recommended daily intake) and high-carbohydrate food during pregnancy have an elevated risk of developing GDM (40).

Analysis of the allele distribution of rs56407701 in the population of the present study suggested a higher frequency of homozygote CC compared with the frequency reported in the 1000 Genomes Project (https://www.internationalgenome. org/). The frequency for homozygote CC controls is $2.5 \%$, according to the 1000 Genomes Project. This may be due to limitations regarding the sample size of the present study. The background of the internal association between these biomarkers (PTPRD gene and miR-450a) and GDM require further study.

In conclusion, the present study provided the first evidence that the SNP rs56407701 in the 3'-UTR of PTPRD was associated with increased susceptibility to GDM. The function the SNP was regulated by miR-450a, which caused suppression of PTPRD expression in patients with the GC and CC genotype. The present study provided evidence that SNPs in this miRNA-binding site may be a novel source of susceptibility loci for human GDM.

\section{Acknowledgements}

Not applicable.

\section{Funding}

No funding received.

\section{Availability of data and materials}

The datasets generated and analyzed during the present study are available from the corresponding author on reasonable request.

\section{Authors' contributions}

YK conducted the experiment, YKand HMH: manuscript writing, literature search and data analysis; HPL and WPS: data analysis and statistical analysis. YK: research design. All authors read and approved the final manuscript

\section{Ethics approval and consent to participate}

The subjects provided written informed consent prior to specimen collection. The study was approved by the Ethics Committee of Qinghai Red-Cross Hospital (Xining, China).

\section{Patient consent for publication}

Not applicable.

\section{Competing interests}

The authors declare that they have no competing interests.

\section{References}

1. Reulen RC, Bright CJ, Winter DL, Fidler MM, Wong K, Guha J, Kelly JS, Frobisher C, Edgar AB, Skinner R, et al: Pregnancy and labor complications in female survivors of childhood cancer: The British childhood cancer survivor study. J Natl Cancer Inst 109: djx056, 2017.

2. Garnaes KK, Mørkved S, Salvesen $\varnothing$ and Moholdt T: Exercise training and weight gain in obese pregnant women: A randomized controlled trial (ETIP Trial). PLoS Med 13: e1002079, 2016.

3. Bao W, Tobias DK, Bowers K, Chavarro J, Vaag A, Grunnet LG, Strøm M, Mills J, Liu A, Kiely M and Zhang C: Physical activity and sedentary behaviors associated with risk of progression from gestational diabetes mellitus to type 2 diabetes mellitus: A prospective cohort study. JAMA Intern Med 174: 1047-1055, 2014.

4. Zhang C, Bao W, Rong Y, Yang H, Bowers K, Yeung E and Kiely M: Genetic variants and the risk of gestational diabetes mellitus: A systematic review. Hum Reprod Update 19: 376-390, 2013.

5. Luoto R, Kinnunen TI, Aittasalo M, Kolu P, Raitanen J, Ojala K, Mansikkamäki K, Lamberg S, Vasankari T, Komulainen T and Tulokas S: Primary prevention of gestational diabetes mellitus and large-for-gestational-age newborns by lifestyle counseling: A cluster-randomized controlled trial. PLoS Med 8: e1001036, 2011.

6. Buchanan TA and Xiang AH: Gestational diabetes mellitus. J Clin Invest 115: 485-491, 2005.

7. Chen L, Magliano DJ and Zimmet PZ: The worldwide epidemiology of type 2 diabetes mellitus-present and future perspectives. Nat Rev Endocrinol 8: 228-236, 2011.

8. Retnakaran R, Ye C, Hanley AJ, Connelly PW, Sermer M, Zinman B and Hamilton JK: Effect of maternal weight, adipokines, glucose intolerance and lipids on infant birth weight among women without gestational diabetes mellitus. CMAJ 184: 1353-1360, 2012.

9. Hou Z, Li M and Cao Y: TCF7L2, CAPN10 polymorphisms are associated with gestational diabetes mellitus (GDM) risks: A meta-analysis. Gynecol Endocrinol 33: 399-404, 2017.

10. Besco JA, Frostholm A, Popesco MC, Burghes AH and Rotter A: Genomic organization and alternative splicing of the human and mouse RPTPrho genes. BMC Genomics 2: 1, 2001.

11. Zhang P, Becka S, Craig SE, Lodowski DT, Brady-Kalnay SM and Wang Z: Cancer-derived mutations in the fibronectin III repeats of PTPRT/PTPrho inhibit cell-cell aggregation. Cell Commun Adhes 16: 146-153, 2009.

12. McAndrew PE, Frostholm A, White RA, Rotter A and Burghes AH: Identification and characterization of RPTP rho, a novel RPTP mu/kappa-like receptor protein tyrosine phosphatase whose expression is restricted to the central nervous system. Brain Res Mol Brain Res 56: 9-21, 1998. 
13. Lee JW, Jeong EG, Lee SH, Nam SW, Kim SH, Lee JY, Yoo NJ and Lee SH: Mutational analysis of PTPRT phosphatase domains in common human cancers. APMIS 115: 47-51, 2007.

14. Yu J, Becka S, Zhang P, Zhang X, Brady-Kalnay SM and Wang Z: Tumor-derived extracellular mutations of PTPRT/PTPrho are defective in cell adhesion. Mol Cancer Res 6: 1106-1113, 2008.

15. Wang D, Cheng Z, Zhao M, Jiao C, Meng Q, Pan H, Xie Y, Li L, Zhu Y, Wang W, et al: PTPN9 induces cell apoptosis by mitigating the activation of Stat 3 and acts as a tumor suppressor in colorectal cancer. Cancer Manag Res 11: 1309-1319, 2019.

16. Chen YT, Lin WD, Liao WL, Lin YJ, Chang JG and Tsai FJ: PTPRD silencing by DNA hypermethylation decreases insulin receptor signaling and leads to type 2 diabetes. Oncotarget 6 : 12997-13005, 2015.

17. Yu H, Wang L, Lv L, Ma C, Du B, Lu T, Jin C, Yan H, Yang Y, Li W, et al: Genome-wide association study suggested the PTPRD polymorphisms were associated with weight gain effects of atypical antipsychotic medications. Schizophr Bull 42: 814-823, 2016.

18. Plasterk RH: Micro RNAs in animal development. Cell 124 877-881, 2006.

19. Valastyan S, Reinhardt F, Benaich N, Calogrias D, Szász AM, Wang ZC, Brock JE, Richardson AL and Weinberg RA: A pleiotropically acting microRNA, miR-31, inhibits breast cancer metastasis. Cell 137: 1032-1046, 2009.

20. Hyun S, Lee JH, Jin H, Nam J, Namkoong B, Lee G, Chung J and Kim VN: Conserved MicroRNA miR-8/miR-200 and its target USH/FOG2 control growth by regulating PI3K. Cell 139: 1096-1108, 2009.

21. Bartel DP: MicroRNAs: Genomics, biogenesis, mechanism, and function. Cell 116: 281-297, 2004.

22. Wang Y, Zhou L, Chen J, Li J, He L, Wu P, Wang M, Tong N, Zhang Z and Fang Y: Association of the 3'UTR FOXO3a polymorphism rs4946936 with an increased risk of childhood acute lymphoblastic leukemia in a Chinese population. Cell Physiol Biochem 34: 325-332, 2014.

23. Wang X, Li W, Ma L, Gao J, Liu J, Ping F and Nie M: Association study of the miRNA-binding site polymorphisms of CDKN2A/B genes with gestational diabetes mellitus susceptibility. Acta Diabetol 52: 951-958, 2015.

24. Liu CJ,Fu X, Xia M,Zhang Q, Gu Z and Guo AY: miRNASNP-v3: A comprehensive database for SNPs and disease-related variations in miRNAs and miRNA targets. Nucleic Acids Res: Sep 29, 2020 (Online ahead of print).

25. Berger H, Crane J, Farine D, Armson A, De La Ronde S, Keenan-Lindsay L, Leduc L, Reid G and Van Aerde J; Maternal-Fetal Medicine Committee; Executive and Coundil fo the Society of Obstetricians and Gynaecologists of Canada: Screening for gestational diabetes mellitus. J Obstet Gynaecol Can 24: 894-912, 2002 (In English, French).

26. Gong J, Tong Y, Zhang HM, Wang K, Hu T, Shan G, Sun J and Guo AY: Genome-wide identification of SNPs in microRNA genes and the SNP effects on microRNA target binding and biogenesis. Hum Mutat 33: 254-263, 2012.

27. Wang Y, Wang L, Yu X and Duan J: Overexpression of miR-450 affects the biological behavior of HepG2 cells by targeting DNMT3a. Onco Targets Ther 12: 5069-5076, 2019.
28. Livak KJ and Schmittgen TD: Analysis of relative gene expression data using real-time quantitative PCR and the 2(-Delta Delta $\mathrm{C}(\mathrm{T})$ ) method. Methods 25: 402-408, 2001

29. Li M, Li F, Lin Q, Shi J, Luo J, Long Q, Yang Q, Ouyang Y, Liu H, Bell RC and Guo J: Cultural adaptation, validation, and primary application of a questionnaire to assess intentions to eat low-glycemic index foods among rural Chinese women. Int J Environ Res Public Health 17: 7577, 2020.

30. Fatima SS, Chaudhry B, Khan TA and Farooq S: KCNQ1 rs2237895 polymorphism is associated with gestational diabetes in Pakistani women. Pak J Med Sci 32: 1380-1385, 2016.

31. Poulsen P, Levin K, Petersen I, Christensen K, Beck-Nielsen $H$ and Vaag A: Heritability of insulin secretion, peripheral and hepatic insulin action, and intracellular glucose partitioning in young and old Danish twins. Diabetes 54: 275-283, 2005.

32. Liu Y, Chai Y, Zhang J and Tang J: A function variant at miR-501 alters susceptibility to hepatocellular carcinoma in a Chinese Han population. Cell Physiol Biochem 38: 2500-2508, 2016.

33. Wei C, Meng L and Zhang Y: miR-450a-5p eliminates MGO-Induced insulin resistance via targeting CREB. Int J Stem Cells 13: 46-54, 2020.

34. Muys BR, Sousa JF, Plaça JR, de Araújo LF, Sarshad AA, Anastasakis DG, Wang X, Li XL, de Molfetta GA, Ramão A, et al: miR-450a acts as a tumor suppressor in ovarian cancer by regulating energy metabolism. Cancer Res 79: 3294-3305, 2019.

35. Luo L, Xie Y, Wang A, Liu X, Xiao F, Zhong X and Zhong C: Desipramine ameliorates $\mathrm{Cr}(\mathrm{VI})$-induced hepatocellular apoptosis via the inhibition of ceramide channel formation and mitochondrial PTP opening. Cell Physiol Biochem 34: 2128-2136, 2014

36. Tsai FJ, Yang CF, Chen CC, Chuang LM, Lu CH, Chang CT, Wang TY, Chen RH, Shiu CF, Liu YM, et al: A genome-wide association study identifies susceptibility variants for type 2 diabetes in Han Chinese. PLoS Genet 6: e1000847, 2010.

37. Below JE, Gamazon ER, Morrison JV, Konkashbaev A, Pluzhnikov A, McKeigue PM, Parra EJ, Elbein SC, Hallman DM, Nicolae DL, et al: Genome-wide association and meta-analysis in populations from Starr County, Texas, and Mexico City identify type 2 diabetes susceptibility loci and enrichment for expression quantitative trait loci in top signals. Diabetologia 54: 2047-2055, 2011.

38. Pei Q, Huang Q, Yang GP, Zhao YC, Yin JY, Song M, Zheng Y, Mo ZH, Zhou HH and Liu ZQ: PPAR- $\gamma 2$ and PTPRD gene polymorphisms influence type 2 diabetes patients' response to pioglitazone in China. Acta Pharmacol Sin 34: 255-261, 2013.

39. Gong K, Qu B, Wang C, Zhou J, Liao D, Zheng W and Pan X: Peroxisome proliferator-activated receptor $\alpha$ facilitates osteogenic differentiation in MC3T3-E1 cells via the sirtuin 1-dependent signaling pathway. Mol Cells 40: 393-400, 2017.

40. Atakora L, Poston L, Hayes L, Flynn AC and White SL: Influence of GDM diagnosis and treatment on weight gain, dietary intake and physical activity in pregnant women with obesity: Secondary analysis of the UPBEAT study. Nutrients 12: 359, 2020. 